\section{Prevalência e fatores associados à obesidade abdominal e ao excesso de peso em adultos maranhenses}

\section{Prevalence and factors associated with abdominal obesity and excess weight among adults from Maranhão, Brazil}

\section{Helma Jane Ferreira Veloso'}

\section{Antônio Augusto Moura da Silva"}

' Programa de Pós-Graduação em Saúde Coletiva da Universidade Federal do Maranhão.

" Departamento de Saúde Pública da Universidade Federal do Maranhão.

Fonte de financiamento: Fundação de Amparo à Pesquisa e ao Desenvolvimento Científico e Tec nológico do Maranhão - FAPEMA. № do processo: PPSUS 4.06-1189/05

Correspondência: Helma Jane Ferreira Veloso, Programa de Pós-Graduação em Saúde Coletiva da Universidade do Maranhão, Rua Barão de Itapary n 155 - Centro, São Luiz, MA CEP 65020-070

E-mail:helma@oi.com.bre aasilva@elo.com.br

\section{Resumo}

Objetivos: Determinar a prevalência de excesso de peso e obesidade abdominal e analisar associações com fatores socioeconômicos, demográficos e comportamentais em adultos maranhenses. Métodos: Amostra probabilística por conglomerados em múltiplos estágios, investigada por meio de inquérito domiciliar com 1.005 adultos de 20 a 59 anos (393 homens e 612 mulheres). Foram aferidos peso, estatura e circunferência da cintura (CC). A obesidade abdominal foi considerada quando $\mathrm{CC} \geq 80$ para mulheres $\mathrm{e} \geq 94$ para homens. Índice de massa corporal (IMC), peso em quilogramas dividido pelo quadrado da altura em metros, $\geq 25 \mathrm{~kg} / \mathrm{m}^{2}$ foi considerado excesso de peso. A variável dependente foi dividida em quatro categorias: IMC e CC normais, excesso de peso sem obesidade abdominal (EP), obesidade abdominal sem excesso de peso (OA) e obesidade abdominal com excesso de peso (OAEP). Foi utilizada a análise de regressão logística multinomial e as estimativas levaram em consideração o delineamento complexo de amostragem. Resultados: Nos homens, a prevalência de OA isolada foi 1,3\%, EP 20,3\% e OAEP 27,5\%. Nas mulheres, 15,5\% tinham OA, $1,3 \%$ EP e $42,0 \%$ apresentavam OAEP ( $p$-valor $<0,001$ ). Na análise multivariável, no modelo para homens, escolaridade $\geq 9$ anos e estar unido foram fatores associados à maior prevalência de EP. Idade $\geq 35$ anos, renda familiar $>2$ salários mínimos e viver com companheira se associaram à maior prevalência de OAEP. Nas mulheres, idade $\geq 35$ anos e viver com companheiro se associaram à maior prevalência de OA. Idade $\geq 35$ anos, viver com companheiro e escolaridade $<9$ anos foram fatores associados à maior prevalência de OAEP. Conclusão: A prevalência de EP foi maior nos homens, enquanto OA predominou nas mulheres. Houve diferença nos fatores associados ao excesso de peso, obesidade abdominal e excesso de peso com obesidade abdominal.

Palavras-chave: Excesso de peso. Obesidade abdominal. Obesidade. Prevalência. Adultos. 


\section{Abstract}

Objective: To determine the prevalence of excess body weight and abdominal obesity and assess the association with socioeconomic, demographic and behavioral factors among adults of Maranhão, Brazil. Methods: Multistage cluster sample investigated through a household survey on 1,005 adults aged 20 to 59 years (393 men and 612 women). Anthropometric measures: weight, height and waist circumference (WC) were assessed. Abdominal obesity was defined as WC $\geq 80$ for women and WC $\geq 94$ for men. Body mass index (BMI), weight in kilograms divided by the square of height in meters, $\geq 25 \mathrm{~kg} / \mathrm{m}^{2}$ was considered excess body weight. The dependent variable was divided into four categories: normal BMI and WC, excess body weight only (EBW), abdominal obesity only (AO) and abdominal obesity with excess body weight (AOEBW). Multinomial logistic regression was used for statistical analysis and estimates took the complex sampling design into account. Results: Among men the prevalence of $\mathrm{AO}$ was $1.3 \%$, EBW $20.3 \%$ and AOEBW $27.5 \%$. Among women $15.5 \%$ presented AO, $1.3 \%$ EBW and $42.0 \%$ AOEBW. There was a significant statistical difference among sexes ( $p$ value $<0.001$ ). In the multivariable analysis, among men, schooling $\geq 9$ years and living with a partner were factors associated with higher prevalence of EBW. Age $\geq 35$ years, family income $>$ two minimum wages and living with a partner were associated with higher prevalence of AOEBW. Among women, age $\geq 35$ and living with a partner were factors associated with AO. Age $\geq 35$ years, living with a partner and schooling $<9$ years were associated with a higher prevalence of AOEBW. Conclusion: the prevalence of EBW was higher among men whereas AO prevailed among women. Factors associated with excess body weight, abdominal obesity and both excess body weight and abdominal obesity varied.

Keywords: Excess weight. Abdominal obesity. Obesity. Prevalence. Adults.

\section{Introdução}

O excesso de peso em adultos, diagnosticado quando o Índice de Massa Corporal (IMC) é maior ou igual a $25 \mathrm{~kg} / \mathrm{m}^{2}$, está associado a outras desordens manifestadas pelo excesso de gordura corporal ${ }^{1}$. As condições mais frequentemente associadas ao excesso de peso são as dificuldades respiratórias, problemas dermatológicos, distúrbios do aparelho locomotor, dislipidemias, doenças cardiovasculares, diabetes tipo II e certos tipos de câncer².

O IMC representa a razão entre o peso em quilogramas dividido pelo quadrado da altura em metros, sendo amplamente utilizado na clínica, mesmo que existam ainda inúmeras restrições teóricas ao uso, pois ele não é capaz de fornecer informações sobre a composição corporal e distribuição da gordura no corpo ${ }^{3}$. É possível que diferenças sexuais, étnicas e nos níveis de atividade física e adiposidade contribuam para algumas das limitações do IMC para a avaliação da obesidade ${ }^{4}$. No entanto, sua facilidade de mensuração, seu amplo uso que viabiliza a comparabilidade e sua alta correlação com a quantidade de gordura corporal são motivos suficientes para a sua utilização, isoladamente ou associado a outros parâmetros antropométricos ${ }^{5}$.

Outro indicador utilizado em estudos epidemiológicos é a medida da Circunferência da Cintura (CC), usada para classificar os diferentes graus de obesidade abdominal. $\mathrm{O}$ primeiro estágio da obesidade abdominal ocorre quando a medida da CC é superior ou igual a $94 \mathrm{~cm}$ nos homens e a $80 \mathrm{~cm}$ nas mulheres ${ }^{6}$. Este acúmulo de gordura na região abdominal é considerado fator de risco para doenças endócrinas, metabólicas e cardiovasculares, mesmo que o IMC esteja dentro dos limites da normalidade ${ }^{7,8}$.

$\mathrm{O}$ excesso de peso entre as mulheres brasileiras tem se mantido praticamente estável. Comparando-se resultados de dois grandes estudos, o excesso de peso reduziu de $40,7 \%$ em 1989 para $39,2 \%$ em 2002; no entanto, neste mesmo período, na população masculina a prevalência de 
excesso de peso aumentou de $29,5 \%$ para $41,0 \%^{9,10}$. A Pesquisa Nacional de Demografia e Saúde (PNDS) de 2006 identificou $52,3 \%$ das mulheres em idade fértil com CC superior ou igual a $80 \mathrm{~cm}$ e $29,8 \%$ superior ou igual a $88 \mathrm{~cm}^{11}$. Não há publicações sobre a prevalência da obesidade abdominal para amostra representativa da população de adultos do sexo masculino no Brasil. Porém, em um estudo feito em Pelotas, Rio Grande do Sul, 61,7\% das mulheres e 37,2\% dos homens apresentaram a CC acima dos padrões normais ${ }^{12}$.

Há escassez de estudos medindo a obesidade abdominal em indivíduos de peso normal e pouco se sabe se os fatores de risco para o excesso de peso e a obesidade abdominal são os mesmos. Diferenças nos fatores de risco entre os sexos também vêm sendo estudadas. Estudos mostram que o excesso de peso está associado à idade superior a 35 anos, a viver com companheiro (a), à baixa escolaridade em ambos os sexos ${ }^{13} \mathrm{e}$ à maior paridade entre as mulheres ${ }^{14}$. Sedentarismo ${ }^{15}$, tabagismo, consumo de bebida alcoólica ${ }^{16}$, idade superior a 40 anos, viver com companheiro e menor renda têm sido associados ao acúmulo de gordura na região abdominal, que também está associado à maior paridade entre as mulheres ${ }^{17}$.

Este trabalho teve por objetivo determinar a prevalência de excesso de peso e obesidade abdominal e analisar associações entre fatores socioeconômicos, demográficos e comportamentais com obesidade abdominal e excesso de peso em adultos residentes nos seis municípios mais populosos do Maranhão.

\section{Métodos}

Foi realizado estudo do tipo transversal, tendo como fonte de dados a pesquisa "Atenção à Saúde no Estado do Maranhão". Nesta pesquisa foram estimadas as prevalências dos principais indicadores de saúde e a utilização de serviços pela população residente nos seis municípios mais populosos do Estado no ano de 2006, em amostra probabilística investigada por meio de inquérito domiciliar ${ }^{18}$.

Foi utilizada amostragem por conglomerados em dois estágios. No primeiro foram selecionados 100 setores censitários dos seis municípios do Estado do Maranhão com mais de cem mil habitantes, com probabilidade proporcional à população a partir da base censitária de 1990 (São Luís, Imperatriz, Caxias, São José de Ribamar, Codó e Timon). No segundo, foi sorteado o quarteirão inicial dentro de cada setor, sendo em seguida sorteado um ponto inicial dentro de cada quarteirão, que foi percorrido até que se entrevistassem 12 adultos em cada setor censitário, não se limitando o número de pessoas incluídas por domicílio. No segundo estágio, a unidade de amostragem foi o indivíduo, mas o processo de amostragem foi realizado em nível de domicílio. Se na última casa houvesse mais adultos do que 0 necessário para se completar 12 pessoas por setor, todos os adultos eram incluídos e, nestes casos, o número de adultos amostrados por setor ultrapassou 12. Como os domicílios foram selecionados com aproximadamente a mesma probabilidade, não houve necessidade de se realizar ponderação. Em $36,6 \%$ dos domicílios foi entrevistado um adulto, em $40,4 \%$ dois adultos, em $13,7 \%$ três adultos, em $7,2 \%$ quatro adultos, em $2,1 \%$ cinco adultos e em $0,2 \%$ seis adultos.

O tamanho amostral foi calculado no STATA 9.0, considerando-se probabilidade de erro do tipo I de $5 \%$, poder estatístico de $90 \%$ e prevalência de excesso de peso de $40 \%$, frequência obtida a partir dos dados da POF (Pesquisa de Orçamento Familiar) 2002-2003 ${ }^{10}$ sendo necessários 1.028 adultos. Neste estudo foram selecionados os indivíduos de 20 a 59 anos de idade, totalizando 1.059 adultos. Deste conjunto, foram excluídos os indivíduos que se recusaram a responder a alguma das perguntas feitas ou quando as respostas foram discrepantes ou incompletas, restando 1.005 indivíduos para análise, correspondendo a 97,8\% da amostra inicialmente calculada.

Foi realizado o exame antropométrico dos entrevistados, medindo-se seu peso, estatura e circunferência da cintura. O 
questionário do adulto abrange questões socioeconômicas, demográficas e comportamentais.

Para obtenção dos dados antropométricos o peso foi medido em quilogramas e aferido em balanças digitais da marca Plenna ${ }^{\circledR}$ com capacidade de $150 \mathrm{~kg}$ e graduação de $100 \mathrm{~g}$, posicionada em local plano. A estatura foi obtida com as pessoas em posição ortostática, descalças, com as pernas e calcanhares juntos, os braços ao longo do corpo e o olhar para frente, sem hiperextensão da coluna. Foi utilizada para mensuração da estatura estadiômetro da marca Alturexata ${ }^{\circledR}$ com graduação de $0,1 \mathrm{~cm}$.

Para a medida da Circunferência da Cintura (CC) foi utilizada fita métrica não flexível marca Sanny®, com precisão de $0,1 \mathrm{~cm}$, colocada diretamente sobre a pele na região mais estreita entre o tórax e o quadril, ou em caso de não haver ponto mais estreito, no ponto médio entre a última costela e a crista ilíaca, sendo a leitura feita no momento da expiração. Os entrevistadores foram treinados e permaneceram sob supervisão, sendo que $10 \%$ das medidas foram repetidas por um segundo examinador para checagem e correções.

$\mathrm{Na}$ análise descritiva, a circunferência da cintura foi categorizada em normal $(<8$ 0 para mulheres e $<94 \mathrm{~cm}$ para homens), risco elevado ( $80 \geq \mathrm{CC}<88$ para mulheres e $94 \geq \mathrm{CC}<102$ para homens), risco muito elevado ( $\geq 88$ para mulheres $\mathrm{e} \geq 102 \mathrm{~cm}$ para homens $)^{6}$. A partir das medidas de peso e estatura foi calculado o Índice de Massa Corpórea - IMC, categorizado da seguinte forma: desnutrido (IMC $\leq 18,4$ ) eutrofia $(18,5 \geq$ IMC $\leq 24,9)$, sobrepeso $(25,0 \geq$ IMC $\leq 29,9)$ e obesidade $(\text { IMC } \geq 30,0)^{19}$.

Neste estudo, a variável dependente foi criada a partir da combinação entre o IMC e a CC, que são os dois indicadores de obesidade mais utilizados em estudos populacionais. A obesidade abdominal foi considerada quando CC $\geq 80 \mathrm{~cm}$ para mulheres e $\geq 94 \mathrm{~cm}$ para homens. O índice de massa corporal (IMC) $\geq 25 \mathrm{~kg} / \mathrm{m}^{2}$ foi considerado excesso de peso. A variável dependente foi dividida em quatro categorias: IMC e CC normais, excesso de peso sem obesidade abdominal (EP), obesidade abdominal sem excesso de peso (OA) e obesidade abdominal com excesso de peso (OAEP). Nas análises a categoria de referência utilizada foi aquela em que os indivíduos apresentavam o estado nutricional menos alterado ou normal tanto pela CC quanto pelo IMC.

Conforme mostrado acima, a variável resposta dispunha de mais de duas categorias, o que exigiu a utilização da análise de Regressão logística multinomial.

A escolaridade, analisada conforme o número de anos completos de estudo, foi categorizada em baixa (oito anos ou menos de estudo) e alta (nove anos ou mais de estudo). O consumo de álcool foi categorizado em: bebeu uma vez ou menos e duas vezes ou mais por semana. A idade foi obtida considerando-se anos completos no momento da entrevista e categorizada em menos de 35 anos e em 35 anos ou mais. A renda familiar, analisada pela quantidade de salários mínimos recebidos pela família por mês, foi classificada em menor ou igual a dois salários mínimos, e em mais de dois salários. O valor do salário mínimo vigente no período da coleta dos dados foi de $\mathrm{R} \$ 350,00$. A situação conjugal foi dicotomizada, considerando-se aqueles que vivem com companheiro(a) e aqueles que vivem sem companheiro(a). $\mathrm{O}$ fumo foi categorizado em fumantes (fuma atualmente pelo menos um cigarro por dia) e não fumantes. A cor da pele foi auto-referida e classificada em branca e não branca. Inserção no mercado de trabalho foi categorizada em sim (aqueles que estavam trabalhando) e não (indivíduos fora da População Economicamente Ativa - PEA).

As análises estatísticas foram realizadas no pacote estatístico Stata, na versão 9,0, utilizando-se o conjunto de comandos svy. $\mathrm{O}$ uso de estimadores convencionais poderia subestimar consideravelmente a variância das estimativas pontuais ${ }^{20}$. O conjunto de comandos svy do Stata levou em consideração o delineamento amostral complexo (amostragem por conglomerados em dois 
estágios e estratificação prévia por município) ${ }^{21}$. A probabilidade de o indivíduo ser sorteado em cada estágio foi calculada. No primeiro estágio esta probabilidade foi obtida multiplicando-se o número de setores amostrados no município pelo número de adultos no setor censitário dividido pela população de adultos de 20 a 59 anos do município. No segundo estágio, a probabilidade foi calculada dividindo-se o número de adultos amostrados no setor pela população do setor censitário. Foi também levado em conta o fato de ter se incluído mais de um indivíduo por domicílio. No comando svyset foram declarados os fatores utilizados para o cálculo das probabilidades de seleção por meio das opções $f p c$. Foram também informados que o cluster no primeiro estágio foi o setor censitário e que, no segundo estágio, o cluster foi o domicílio. Na opção strata foi declarado que a amostra foi estratificada por município. Foram calculados intervalos de confiança de $95 \%$ das estimativas de prevalência e o efeito de desenho para as variáveis do estudo.

Inicialmente foi realizada a análise descritiva e posteriormente foi feita a análise univariável, utilizando-se a regressão logística multinomial simples, com estimativa da razão de prevalências não ajustada e intervalo de confiança de 95\% (IC95\%). Em seguida procedeu-se à análise multivariável por meio de regressão logística multinomial múltipla. As variáveis independentes que apresentaram significância $<0,20(P<$ 0,20 ) foram consideradas como candidatas ao modelo final, mas só permaneceram aquelas que apresentaram nível de significância inferior a 0,10 para se obter melhor controle do confundimento. Considerou-se alfa $\leq 0,05$.

A pesquisa atende aos critérios da Resolução 196/96 do Conselho Nacional de Saúde e suas complementares e todos os entrevistados assinaram o Termo de Consentimento Livre e Esclarecido. A pesquisa foi aprovada pelo Comitê de Ética em Pesquisa do Hospital Universitário da UFMA parecer $\mathrm{N}^{\circ} 275 / 06$, não havendo conflito de interesses.

\section{Resultados}

A prevalência de desnutrição foi de $4,3 \%$ entre os homens e $6,4 \%$ entre as mulheres. Dos homens, 31,1\% apresentaram sobrepeso, o que foi observado em $26,8 \%$ das mulheres. A prevalência de obesidade foi de $13,0 \%$ entre os homens e de $15,3 \%$ entre as mulheres. Não houve diferença estatisticamente significante no estado nutricional, avaliado pelo IMC, entre os dois sexos. De acordo com a CC, $17,5 \%$ dos homens e $26,0 \%$ das mulheres apresentaram risco elevado, e $11,2 \%$ dos homens e $31,5 \%$ das mulheres apresentaram risco muito elevado. Nos homens, a prevalência de OA isolada foi $1,3 \%$, EP $20,3 \%$ e OAEP $27,5 \%$. Nas mulheres, $15,5 \%$ tinham OA isolada, $1,3 \%$ apenas EP e 42,0\% apresentavam OAEP. Houve diferença estatisticamente significante entre os sexos ( $p$-valor $<0,001$ ) (Tabela 1).

A faixa etária mais observada foi de adultos com menos de 35 anos de idade. Mais de $28 \%$ dos homens e $6,9 \%$ das mulheres bebiam duas ou mais vezes na semana. A cor branca foi referida por $22,6 \%$ dos homens e $23,5 \%$ das mulheres. Renda familiar superior a dois salários mínimos foi referida por $55,0 \%$ dos homens e $53,0 \%$ das mulheres. A prevalência de fumantes foi de $20,9 \%$ entre os homens e $11,8 \%$ entre as mulheres. O percentual de homens e mulheres que viviam sem companheiro(a) foi de $37,9 \%$ e $43,1 \%$, respectivamente. Cinquenta e oito por cento dos homens e mulheres tinham nove anos ou mais de estudo. A percentagem de homens e mulheres fora da População Economicamente Ativa (PEA) foi de 14,8\% e $47,5 \%$, respectivamente. Os efeitos de desenho foram baixos para todas as variáveis, sendo o mais elevado para o consumo de álcool - 1,359 (Tabela 1).

Na Tabela 2 tem-se a análise não ajustada dos fatores associados ao excesso de peso e a obesidade abdominal nos homens. Idade $\geq 35$ anos esteve associada à obesidade abdominal isolada $(\mathrm{RP}=7,26)$. Maior escolaridade (9 anos ou mais de estudo) esteve associada à maior prevalência de 
Tabela 1 - Caracterização dos adultos residentes nos seis municípios mais populosos do Estado do Maranhão em 2006. Table 1 - Description of adults living in the six most populated municipalities in the state of Maranhão in 2006.

\begin{tabular}{|c|c|c|c|c|c|c|c|c|}
\hline \multirow[t]{2}{*}{ Variáveis } & \multicolumn{3}{|c|}{ Masculino $(n=393)$} & \multicolumn{3}{|c|}{ Feminino $(n=612)$} & \multirow{2}{*}{ p-valor * } & \multirow{2}{*}{$\begin{array}{c}\text { Efeito } \\
\text { do } \\
\text { desenho }\end{array}$} \\
\hline & $\mathrm{N}$ & $\%$ & $\begin{array}{c}\text { Intervalo de } \\
\text { Confiança de } 95 \%\end{array}$ & $\mathrm{~N}$ & $\%$ & $\begin{array}{c}\text { Intervalo de } \\
\text { Confiança de } 95 \%\end{array}$ & & \\
\hline Idade (anos) & & & & & & & 0,949 & 0,736 \\
\hline$<35$ & 208 & 52,9 & $(48,0 ; 57,7)$ & 325 & 53,1 & $(49,2 ; 56,8)$ & & \\
\hline$\geq 35$ & 185 & 47,1 & $(42,2 ; 51,9)$ & 287 & 46,9 & $(43,1 ; 50,7)$ & & \\
\hline $\begin{array}{l}\text { Consumo de álcool } \\
\text { (vezes por semana) }\end{array}$ & & & & & & & $<0,001$ & 1,359 \\
\hline$\leq 1$ & 282 & 71,8 & $(66,6 ; 76,3)$ & 570 & 93,1 & $(90,5 ; 95,0)$ & & \\
\hline$\geq 2$ & 111 & 28,2 & $(23,3 ; 33,3)$ & 42 & 6,9 & $(4,9 ; 9,4)$ & & \\
\hline Estado nutricional & & & & & & & 0,093 & 1,093 \\
\hline Desnutrição & 17 & 4,3 & $(2,4 ; 7,5)$ & 39 & 6,4 & $(4,5 ; 8,9)$ & & \\
\hline Peso normal & 191 & 48,6 & $(42,9 ; 54,2)$ & 315 & 51,5 & $(47,5 ; 55,4)$ & & \\
\hline Sobrepeso & 134 & 34,1 & $(29,3 ; 39,1)$ & 164 & 26,8 & $(23,5 ; 30,3)$ & & \\
\hline Obesidade & 51 & 13,0 & $(9,9 ; 16,8)$ & 94 & 15,3 & $(12,5 ; 18,7)$ & & \\
\hline Circunferência da Cintura & & & & & & & $<0,001$ & 0,770 \\
\hline Normal & 280 & 71,3 & $(66,1 ; 75,8)$ & 260 & 42,5 & $(38,8 ; 46,2)$ & & \\
\hline Risco & 69 & 17,5 & $(14,3 ; 21,3)$ & 159 & 26,0 & $(22,5 ; 29,7)$ & & \\
\hline Alto risco & 44 & 11,2 & $(8,4 ; 14,6)$ & 193 & 31,5 & $(27,7 ; 35,6)$ & & \\
\hline $\begin{array}{l}\text { Circunferência da cintura } \\
\text { e índice de massa corporal }\end{array}$ & & & & & & & $<0,001$ & 1,014 \\
\hline Normais & 200 & 50,9 & $(45,3 ; 56,4)$ & 252 & 41,2 & $(37,5 ; 44,8)$ & & \\
\hline Obesidade abdominal & 5 & 1,3 & $(0,4 ; 3,5)$ & 95 & 15,5 & $(12,6 ; 18,9)$ & & \\
\hline Excesso de peso & 80 & 20,3 & $(16,6 ; 24,7)$ & 8 & 1,3 & $(0,6 ; 2,5)$ & & \\
\hline $\begin{array}{l}\text { Obesidade abdominal } \\
\text { e excesso de peso }\end{array}$ & 108 & 27,5 & $(23,0 ; 32,4)$ & 257 & 42,0 & $(38,1 ; 45,9)$ & & \\
\hline Cor da pele & & & & & & & 0,708 & 0,735 \\
\hline Branca & 89 & 22,6 & $(18,6 ; 27,2)$ & 144 & 23,5 & $(20,2 ; 27,1)$ & & \\
\hline Não branca & 304 & 77,4 & $(72,8 ; 81,3)$ & 468 & 76,5 & $(72,8 ; 79,1)$ & & \\
\hline $\begin{array}{l}\text { Renda familiar (salários } \\
\text { mínimos) }\end{array}$ & & & & & & & 0,474 & 0,639 \\
\hline$\leq 2$ & 177 & 45,0 & $(37,9 ; 52,3)$ & 288 & 47,0 & $(40,9 ; 52,9)$ & & \\
\hline$>2$ & 216 & 55,0 & $(47,6 ; 62,0)$ & 324 & 53,0 & $(47,0 ; 59,0)$ & & \\
\hline Fumo & & & & & & & $<0,001$ & 1,011 \\
\hline Não fumantes & 311 & 79,1 & $(74,3 ; 83,2)$ & 540 & 88,2 & $(85,0 ; 90,8)$ & & \\
\hline Fumantes & 82 & 20,9 & $(16,7 ; 25,6)$ & 72 & 11,8 & $(9,1 ; 14,9)$ & & \\
\hline Situação Conjugal & & & & & & & 0,036 & 0,578 \\
\hline Com companheiro/a & 244 & 62,1 & $(57,2 ; 66,6)$ & 348 & 56,9 & $(52,1 ; 61,4)$ & & \\
\hline Sem companheiro/a & 149 & 37,9 & $(33,3 ; 42,7)$ & 264 & 43,1 & $(38,5 ; 47,8)$ & & \\
\hline Escolaridade (anos de estudo) & & & & & & & 0,998 & 0,735 \\
\hline$\leq 8$ & 165 & 42,0 & $(36,1 ; 48,0)$ & 257 & 42,0 & $(36,8 ; 47,3)$ & & \\
\hline$\geq 9$ & 228 & 58,0 & $(51,9 ; 63,8)$ & 355 & 58,0 & $(52,7 ; 63,1)$ & & \\
\hline $\begin{array}{l}\text { Inserção no mercado de } \\
\text { trabalho }\end{array}$ & & & & & & & $<0,001$ & 1,022 \\
\hline Sim & 335 & 85,2 & $(81,0 ; 88,6)$ & 321 & 52,5 & $(47,8 ; 56,9)$ & & \\
\hline Não & 58 & 14,8 & $(11,3 ; 18,9)$ & 291 & 47,5 & $(43,0 ; 52,1)$ & & \\
\hline
\end{tabular}

* Qui-quadrado de Person/Person's chi-square test 
$\mathrm{EP}(\mathrm{RP}=1,99)$. Viver sem companheiro(a) esteve associado à menor prevalência de $\mathrm{EP}(\mathrm{RP}=0,37)$ e de OAEP $(\mathrm{RP}=0,22)$ entre os homens. A idade superior a 35 anos foi associada à maior prevalência de OAEP (RP $=3,78$ ). A renda familiar superior a dois salários mínimos foi associada à maior prevalência de OAEP $(\mathrm{RP}=2,12)$. Como não havia nenhum homem fora da PEA com OA isolada, não foi possível inserir a variável inserção no mercado de trabalho no modelo masculino.
Na Tabela 3 tem-se a análise não ajustada dos fatores associados ao excesso de peso e a obesidade abdominal nas mulheres. A escolaridade superior a nove anos de estudo esteve associada à menor prevalência de $\mathrm{OA}(\mathrm{RP}=0,58)$ e de OAEP $(\mathrm{RP}=0,44)$. Idade superior a 35 anos foi associada à maior prevalência de $\mathrm{OA}(\mathrm{RP}=3,73)$ e de OAEP $(\mathrm{RP}=4,08)$. Viver sem companheiro esteve associado à menor prevalência de $\mathrm{AO}(\mathrm{RP}=$ $0,27)$ e de OAEP $(\mathrm{RP}=0,43)$.

$\mathrm{Na}$ análise ajustada para o sexo mas-

Tabela 2 -Análise não ajustada dos fatores associados à obesidade abdominal e ao excesso de peso em homens maranhenses, 2006.

Table 2 - Non-adjusted analysis of factors associated with abdominal obesity and excess weight among men from Maranhão, 2006.

\begin{tabular}{|c|c|c|c|c|c|c|}
\hline \multirow[b]{2}{*}{ Variáveis (n=393) } & \multicolumn{2}{|c|}{ Obesidade abdominal } & \multicolumn{2}{|c|}{ Excesso de peso } & \multicolumn{2}{|c|}{$\begin{array}{c}\text { Obesidade abdominal com } \\
\text { excesso de peso }\end{array}$} \\
\hline & $\mathrm{RP}^{*}(\mathrm{IC} 95 \%)$ & $\mathrm{p}+$ & RP* (IC 95\%) & $p+$ & RP* (IC 95\%) & $\mathrm{p} \dagger$ \\
\hline $\begin{array}{l}\text { Escolaridade } \\
\text { (anos de estudo) }\end{array}$ & & 0,764 & & 0,014 & & 0,249 \\
\hline$\leq 8$ & 1 & & 1 & & 1 & \\
\hline$\geq 9$ & $1,35(0,18 ; 10,21)$ & & $1,99(1,15 ; 3,43)$ & & $1,36(0,80 ; 2,33)$ & \\
\hline $\begin{array}{l}\text { Consumo de álcool } \\
\text { (vezes por semana) }\end{array}$ & & 0,692 & & 0,653 & & 0,661 \\
\hline$\leq 1$ & 1 & & 1 & & 1 & \\
\hline$\geq 2$ & $0,62(0,06 ; 6,44)$ & & $1,14(0,63 ; 2,03)$ & & $0,87(0,48 ; 1,58)$ & \\
\hline Idade (anos) & & 0,030 & & 0,097 & & $<0,001$ \\
\hline$<35$ & 1 & & 1 & & 1 & \\
\hline$\geq 35$ & $7,26(1,21 ; 43,47)$ & & $1,58(0,92 ; 2,65)$ & & $3,78(2,29 ; 6,25)$ & \\
\hline $\begin{array}{l}\text { Renda familiar } \\
\text { (salários mínimos) }\end{array}$ & & 0,626 & & 0,138 & & 0,002 \\
\hline$\leq 2$ & 1 & & 1 & & 1 & \\
\hline$>2$ & $1,65(0,21 ; 12,91)$ & & $1,57(0,86 ; 2,87)$ & & $2,12(1,31 ; 3,41)$ & \\
\hline Situação Conjugal & & 0,215 & & 0,001 & & $<0,001$ \\
\hline Com companheira & 1 & & 1 & & 1 & \\
\hline Sem companheira & $0,23(0,02 ; 2,38)$ & & $0,37(0,21 ; 0,66)$ & & $0,22(0,13 ; 0,37)$ & \\
\hline Fumo & & 0,297 & & 0,298 & & 0,373 \\
\hline Não fumante & 1 & & 1 & & 1 & \\
\hline Fumante & $2,23(0,48 ; 10,21)$ & & $0,71(0,37 ; 1,35)$ & & $0,76(0,41 ; 1,39)$ & \\
\hline Cor da pele & & 0,221 & & 0,472 & & 0,693 \\
\hline Branca & 1 & & 1 & & 1 & \\
\hline Não branca & $0,39(0,09 ; 1,75)$ & & $0,79(0,42 ; 1,48)$ & & $0,88(0,47 ; 1,65)$ & \\
\hline
\end{tabular}

* RP= Razão de Prevalências (IC-intervalo de confiança de 95\%) estimada pelo modelo de regressão logística multinomial simples/Prevalence Ratio (95\% confidence interval) estimated by simple multinomial logistic regression model

t $\mathrm{p}$-valor do teste log-likelihood/p value for the log-likelihood test 
Tabela 3 -Análise não ajustada dos fatores associados à obesidade abdominal e ao excesso de peso em mulheres maranhenses, 2006.

Table 3 - Non-adjusted analysis of factors associated with abdominal obesity and excess weight among women from Maranhão, 2006.

\begin{tabular}{|c|c|c|c|c|c|c|}
\hline \multirow[b]{2}{*}{ Variáveis(n=612) } & \multicolumn{2}{|c|}{ Obesidade abdominal } & \multicolumn{2}{|c|}{ Excesso de peso } & \multicolumn{2}{|c|}{$\begin{array}{c}\text { Obesidade abdominal com } \\
\text { excesso de peso }\end{array}$} \\
\hline & $\mathrm{RP}^{*}(\mathrm{IC} 95 \%)$ & $\mathrm{p}+$ & $\mathrm{RP}^{*}(\mathrm{IC} 95 \%)$ & $\mathrm{p}+$ & $\mathrm{RP}^{*}(\mathrm{IC} 95 \%)$ & $\mathrm{p}+$ \\
\hline Escolaridade (anos de estudo) & & 0,036 & & 0,290 & & $<0,001$ \\
\hline$\leq 8$ anos & 1 & & 1 & & 1 & \\
\hline$\geq 9$ anos & $0,58(0,35 ; 0,96)$ & & $0,46(0,11 ; 1,94)$ & & $0,44(0,31 ; 0,64)$ & \\
\hline $\begin{array}{l}\text { Consumo de álcool (vezes por } \\
\text { semana) }\end{array}$ & & 0,248 & & 0,116 & & 0,399 \\
\hline$\leq 1$ & 1 & & 1 & & 1 & \\
\hline$\geq 2$ & $0,50(0,16 ; 1,61)$ & & $3,86(0,71 ; 21,04)$ & & $0,77(0,41 ; 1,41)$ & \\
\hline Idade (anos) & & $<0,001$ & & 0,184 & & $<0,001$ \\
\hline$<35$ & 1 & & 1 & & 1 & \\
\hline$\geq 35$ & $3,73(2,16 ; 6,43)$ & & $2,6(0,63 ; 10,72)$ & & $4,08(2,73 ; 6,10)$ & \\
\hline Renda familiar (salários mínimos) & & 0,921 & & 0,353 & & 0,585 \\
\hline$\leq 2$ & 1 & & 1 & & 1 & \\
\hline$>2$ & $0,97(0,56 ; 1,68)$ & & $0,50(0,11 ; 2,16)$ & & $0,91(0,66 ; 1,24)$ & \\
\hline Situação Conjugal & & $<0,001$ & & 0,734 & & $<0,001$ \\
\hline Com companheiro & 1 & & 1 & & 1 & \\
\hline Sem companheiro & $0,27(0,16 ; 0,46)$ & & $1,29(0,29 ; 5,71)$ & & $0,43(0,30 ; 0,61)$ & \\
\hline Fumo & & 0,771 & & 0,278 & & 0,939 \\
\hline Não fumante & 1 & & 1 & & 1 & \\
\hline Fumante & $1,11(0,54 ; 2,28)$ & & $2,56(0,46 ; 14,2)$ & & $0,97(0,54 ; 1,73)$ & \\
\hline Cor da pele & & 0,817 & & 0,422 & & 0,365 \\
\hline Branca & 1 & & 1 & & 1 & \\
\hline Não branca & $1,06(0,61 ; 1,83)$ & & $2,38(0,28 ; 20,25)$ & & $1,22(0,78 ; 1,89)$ & \\
\hline Inserção no mercado de trabalho & & 0,967 & & 0,200 & & 0,411 \\
\hline Sim & 1 & & 1 & & 1 & \\
\hline Não & $1,01(0,60 ; 1,69)$ & & $0,34(0,06 ; 1,77)$ & & $0,87(0,63 ; 1,20)$ & \\
\hline
\end{tabular}

* RP= Razão de Prevalências (IC - intervalo de confiança de 95\%) estimada pelo modelo de regressão logística multinomial simples / Prevalence Ratio (95\% confidence interval) estimated by simple multinomial logistic regression model

† p-valor do teste log-likelihood / $p$ value for the log-likelihood test

culino, a idade $\geq 35$ anos $(\mathrm{RP}=2,87)$, renda familiar $>2$ salários $(\mathrm{RP}=2,00)$ e viver sem companheira $(\mathrm{RP}=0,29)$ permaneceram independentemente associadas à OAEP. Escolaridade $\geq 9$ anos $(\mathrm{RP}=2,17)$ e viver sem companheira $(\mathrm{RP}=0,34)$ permaneceram independentemente associadas ao EP. Nenhuma variável permaneceu associada à obesidade abdominal (Tabela 4).

$\mathrm{Na}$ análise ajustada para o sexo femini- no, idade $\geq 35$ anos $(\mathrm{RP}=3,53)$ foi associada à maior prevalência, e viver sem companheiro $(\mathrm{RP}=0,28)$ à menor prevalência de OA. Nenhuma das variáveis mostrou associação estatisticamente significante com o EP isolado entre as mulheres. Escolaridade $\geq$ 9 anos $(\mathrm{RP}=0.53)$ e viver sem companheiro $(\mathrm{RP}=0.44)$ foram associados à menor prevalência, e a idade $\geq 35$ anos $(\mathrm{RP}=3.79)$ à maior prevalência de OAEP (Tabela 5). 
Tabela 4 - Análise ajustada dos fatores associados à obesidade abdominal e ao excesso de peso em homens maranhenses, 2006.

Table 4 - Adjusted analysis of factors associated with abdominal obesity and excess weight among men from Maranhão, 2006.

\begin{tabular}{|c|c|c|c|c|}
\hline \multirow[b]{2}{*}{ Variáveis $(n=393)$} & \multicolumn{2}{|c|}{ Excesso de peso } & \multicolumn{2}{|c|}{$\begin{array}{l}\text { Obesidade abdominal com } \\
\text { excesso de peso }\end{array}$} \\
\hline & $\mathrm{RP} *(\mathrm{IC} 95 \%)$ & $p+$ & $\mathrm{RP}^{*}(\mathrm{IC} 95 \%)$ & $\mathrm{p} \dagger$ \\
\hline $\begin{array}{l}\text { Escolaridade } \\
\text { (anos de estudo) }\end{array}$ & & 0,021 & & \\
\hline$\leq 8$ & 1 & & - & \\
\hline$\geq 9$ & $2,17(1,12 ; 4,17)$ & & - & \\
\hline Idade & & & & 0.001 \\
\hline$<35$ & - & & 1 & \\
\hline$\geq 35$ & _- & & $2,87(1,55 ; 5,31)$ & \\
\hline $\begin{array}{l}\text { Renda familiar } \\
\text { (salários mínimos) }\end{array}$ & & & & 0,008 \\
\hline$\leq 2$ & - & & 1 & \\
\hline$\geq 2$ & _- & & $2,00(1,21 ; 3,33)$ & \\
\hline Situação Conjugal & & 0,001 & & $<0,001$ \\
\hline Com companheira & 1 & & 1 & \\
\hline Sem companheira & $0,34(0,18 ; 0,65)$ & & $0,29(0,16 ; 0,52)$ & \\
\hline
\end{tabular}

* RP= Razão de Prevalências (intervalo de confiança de 95\%) estimada pelo modelo de regressão logística multinomial múltipla/Prevalence Ratio (95\% confidence interval) estimated by multiple multinomial logistic regression model † $\mathrm{p}$-valor do teste log-likelihood/p value for the log-likelihood test

Tabela 5 - Análise ajustada dos fatores associados à obesidade abdominal e ao excesso de peso em mulheres maranhenses, 2006.

Table 5 - Adjusted analysis of factors associated with abdominal obesity and excess weight among women from Maranhão, 2006.

\begin{tabular}{|c|c|c|c|c|}
\hline \multirow[b]{2}{*}{ Variáveis $(\mathrm{n}=612)$} & \multicolumn{2}{|c|}{ Obesidade abdominal } & \multicolumn{2}{|c|}{$\begin{array}{l}\text { Obesidade abdominal com } \\
\text { excesso de peso }\end{array}$} \\
\hline & $\mathrm{RP}^{*}(\mathrm{IC} 95 \%)$ & $\mathrm{p}$-valor $†$ & RP* (IC 95\%) & p-valor † \\
\hline $\begin{array}{l}\text { Escolaridade } \\
\text { (anos de estudo) }\end{array}$ & & & & 0,009 \\
\hline$\leq 8$ & - & & 1 & \\
\hline$\geq 9$ & - & & $0,53(0,34 ; 0,85)$ & \\
\hline Idade (anos) & & $<0,001$ & & $<0,001$ \\
\hline$<35$ & 1 & & 1 & \\
\hline$\geq 35$ & $3,53(1,99 ; 6,24)$ & & $3,79(2,41 ; 5,93)$ & \\
\hline Situação Conjugal & & $<0,001$ & & $<0,001$ \\
\hline Com companheiro & 1 & & 1 & \\
\hline Sem companheiro & $0,28(0,16 ; 0,43)$ & & $0,44(0,30 ; 0,64)$ & \\
\hline
\end{tabular}




\section{Discussão}

A prevalência de excesso de peso isolado foi maior nos homens, enquanto a obesidade abdominal predominou nas mulheres. Houve diferença nos fatores associados ao excesso de peso, obesidade abdominal e obesidade abdominal acompanhada de excesso de peso, e também foram detectadas variações de acordo com o sexo. Menor escolaridade esteve associada à maior prevalência de OAEP, mas não de OA em mulheres. Maior escolaridade associou-se à maior prevalência de EP em homens. Viver com companheiro esteve associado à maior prevalência de OA e OAEP em mulheres e de EP e OAEP em homens. Idade $\geq 35$ anos foi associada à maior prevalência de $\mathrm{OA}$ em mulheres e de OAEP em ambos os sexos.

Os resultados deste estudo foram obtidos a partir de uma amostra representativa da população de adultos, de ambos os sexos, dos seis municípios mais populosos do Estado do Maranhão, localizado no Nordeste do Brasil. O percentual de perdas $(2,2 \%)$ foi muito baixo, possibilitando a generalização dos resultados. Além de ser um estudo de base populacional, este trabalho incorporou a circunferência da cintura, medida pouco utilizada em estudos populacionais, para diagnóstico de obesidade abdominal entre adultos de uma das regiões mais pobres do país.

Deve-se considerar como ponto forte deste estudo o fato de a análise estatística ter sido realizada com o desfecho criado a partir da combinação entre os dois parâmetros antropométricos mais aconselhados em estudos populacionais. A combinação entre estes dois parâmetros possibilitou a observação de alta prevalência de obesidade abdominal em mulheres que tinham IMC normal, o que concorda com alguns estudos que mostram que o IMC tem baixa sensibilidade para a identificação de excesso de gordura corporal. Yao et al. observaram que mais de $30 \%$ de indivíduos classificados como eutróficos pelo IMC apresentaram excesso de gordura corporal ${ }^{3}$. Outro estudo identificou que $61 \%$ das mulheres e $23,6 \%$ dos homens com IMC $<30 \mathrm{~kg} / \mathrm{m}^{2}$ apresentaram percentual de gordura corporal medido por bioimpedância maior que $30 \%$ e maior que $25 \%$, respectivamente ${ }^{22}$.

Uma das limitações do estudo foi ter incluído maior número de mulheres na amostra, o que pode ser explicado pela maior dificuldade de se encontrar homens no domicílio, já que estes representavam, em maior percentual, a população economicamente ativa.

Neste estudo não houve diferença estatisticamente significante no excesso de peso e na obesidade segundo sexo, de acordo com a avaliação feita pelo IMC. Os dados da Pesquisa de Orçamentos Familiares (POF) realizada nos anos de 2002-2003 não mostraram diferenças substanciais na prevalência do excesso de peso entre os sexos. Já a obesidade foi menos prevalente nos homens do que nas mulheres, com diferença estatisticamente significante ${ }^{10}$.

De acordo com a classificação de excesso de gordura na região abdominal, medida pela CC, a prevalência de obesidade abdominal entre as mulheres foi o dobro daquela encontrada entre os homens. Em outro estudo sobre o potencial diagnóstico da obesidade centralizada, as mulheres tiveram sete vezes mais chance de apresentar obesidade abdominal do que os homens ${ }^{23}$.

$\mathrm{Na}$ análise multivariável para o sexo masculino nenhuma das variáveis explanatórias esteve associada à OA isolada. Já nas mulheres, nenhuma das variáveis explicou o EP isolado. Este resultado pode ser explicado pelo reduzido número de homens e mulheres que se enquadraram nestas categorias.

Menor escolaridade esteve associada à maior prevalência de OAEP em mulheres, enquanto maior escolaridade associou-se à maior prevalência de EP em homens. No estudo Pró-Saúde, feito na Universidade Estadual do Rio de Janeiro, a escolaridade mostrou forte associação inversa com o IMC entre as mulheres e não foi observada associação entre os homens ${ }^{24}$. No entanto, no presente estudo, a maior escolaridade esteve associada ao excesso de peso isolado 
apenas entre os homens. A associação de menor escolaridade com excesso de peso entre as mulheres foi observada apenas quando havia obesidade abdominal e excesso de peso (OAEP). Em investigação feita no Brasil, a baixa escolaridade mostrou-se associada à obesidade entre as mulheres, enquanto nos homens a obesidade predominou naqueles de maior escolaridade ${ }^{25}$, corroborando os resultados apresentados neste estudo.

Renda familiar maior que dois salários mínimos esteve associada à maior prevalência de OAEP entre os homens. Resultados semelhantes também foram observados em estudo feito na zona urbana de Pelotas, onde a distribuição de gordura abdominal esteve associada à maior renda familiar entre os homens ${ }^{17}$. Em estudo populacional realizado com adultos brasileiros foi observado que as mulheres que viviam nas regiões mais pobres do país tinham maior chance de apresentar obesidade ${ }^{25}$. No entanto, neste estudo a renda não explicou nem a obesidade abdominal nem o excesso de peso entre as mulheres, fato que merece destaque já que o Maranhão é um dos Estados mais pobres do país. Foi observada baixa variabilidade na renda dos pesquisados, principalmente entre as mulheres, o que pode ter contribuído para a ausência de associação da renda com os desfechos.

A variável inserção no mercado de trabalho não mostrou associação com os desfechos nas mulheres, divergindo dos resultados encontrados em estudo realizado no sul do Brasil, em que estar trabalhando mostrou ser fator de proteção para a obesidade abdominal em mulheres. A metodologia utilizada para analisar a inserção no mercado de trabalho foi igual nos dois estudos, não sendo considerada a atividade que as mulheres exerciam e sim o fato de estarem trabalhando fora do $\operatorname{lar}^{26}$.

Considerando-se as variáveis socioeconômicas (escolaridade, renda e inserção no mercado de trabalho), a escolaridade teve maior influência, pois foi associada aos desfechos nos dois sexos. No entanto, a alta escolaridade esteve associada à menor prevalência de OAEP entre as mulheres, enquanto nos homens a alta escolaridade mostrou-se associada à maior prevalência de EP. Resultados semelhantes foram descritos em estudos anteriores, levantando a hipótese de que mulheres com maior nível de escolaridade têm maior preocupação em manter/perder peso pelo ideal estético e valorização social da magreza, sendo mais motivadas do que os homens a alcançar o estereótipo da figura esbelta ${ }^{27,28}$. Há uma tendência cultural a considerar a magreza como uma situação ideal de aceitação social para mulheres ${ }^{29,30}$. Em contrapartida, nos homens ocorre a tendência a se acatar, como ideal, um corpo mais forte ou mais volumoso ${ }^{31}$.

Idade maior ou igual a 35 anos esteve associada à maior prevalência de $\mathrm{OA}$ e de OAEP entre as mulheres. Em estudo feito com mulheres no Rio de Janeiro, a idade perdeu a associação estatisticamente significante com a obesidade abdominal quando foi feito o ajuste pelas categorias de IMC $^{32}$. Entre os homens, ter 35 anos ou mais de idade esteve associado à maior prevalência de OAEP, mas não explicou o EP ou a OA isolados. Em outro estudo realizado em Campos-RJ, a idade mais elevada foi considerada fator de risco para a obesidade entre os homens ${ }^{33}$.

Nenhum dos fatores comportamentais analisados neste estudo mostrou associação com os desfechos, corroborando os resultados apresentados em estudo realizado em Belo Horizonte ${ }^{27}$. No entanto, outros estudos mostraram associação inversa entre fumo e obesidade abdominal ${ }^{17,33,34}$. A prevalência estimada de tabagismo foi baixa no presente estudo, podendo ter contribuído para a não associação com os desfechos. No caso do consumo de álcool semanal, os resultados mostrados aqui são consistentes com outros trabalhos, que também falharam em demonstrar associação entre o consumo de álcool e a obesidade abdominal ${ }^{2,16,17}$. Interpretar os resultados das variáveis comportamentais em um estudo transversal é difícil devido à possibilidade de causalidade reversa, sendo este fato uma 
limitação do estudo. Outra limitação se deveu ao fato da variável atividade física não ter sido incluída na análise por se tratar de estudo transversal, onde é possível ocorrer causalidade reversa. Além do mais, optou-se por não incluir ajuste para atividade física, pois esta é possível variável mediadora da associação entre escolaridade e obesidade.

A cor da pele não explicou o EP isolado, OA isolada ou a OAEP em homens ou mulheres corroborando outros estudos conduzidos no Brasil ${ }^{12,17,26}$.

Os dados deste estudo revelam que viver sem companheiro(a) está associado à menor prevalência de $\mathrm{OA}$ em mulheres, EP entre os homens e OAEP em ambos os sexos. Este resultado se assemelha ao encontrado em estudo feito em São Leopoldo com mulheres de 20-60 anos, em que estar casada ou ser viúva foi considerado fator de risco para obesidade abdominal ${ }^{26}$. Em outro estudo, realizado em Pelotas, estar casado ou viver com companheiro(a) foi fator de risco para obesidade abdominal em ambos os sexos ${ }^{12}$. No entanto, os resultados do modelo final de regressão logística hierarquizado não mostraram associação entre situação conjugal e obesidade abdominal em mulheres usuárias do Sistema Único de Saúde (SUS), residentes na região metropolitana de Belo Horizonte, Brasil ${ }^{27}$.

Foi observada alta prevalência de obesidade abdominal em mulheres com IMC normal. Considerando os males que a obesidade abdominal pode acarretar ao indivíduo, a CC deveria ser medida em conjunto com o IMC na prática clínica, especialmente nas mulheres, pois isto possibilitaria o diagnóstico de indivíduos em risco nutricional precocemente. Existe a necessidade de implantação de medidas de controle da obesidade abdominal e total, com foco nas mulheres que vivem em união, têm 35 anos de idade ou mais e possuem baixo nível de escolaridade. Houve baixa prevalência de obesidade abdominal entre os homens, sendo evidente apenas quando associada ao excesso de peso. Para homens, a atenção deve ser direcionada àqueles com idade igual ou superior a 35 anos, com renda e escolaridade elevadas e que possuem companheira.

\section{Referências}

1. Carvalho KMB. Obesidade. In: Cuppari L (ed.). Nutrição: nutrição clínica do adulto. Barueri: Manole; 2002. p. 13150 .

2. WHO. Obesity: Preventing, and Managing the Global Epidemic. Geneva: World Health Organization WHO Consultation on Obesity; 1998.

3. Yao M, Roberts SB, Ma G, Pan H, McCrory MA. Field methods for body composition assessment are valid in healthy Chinese adults. J Nutr 2002; 2: 310-7.

4. Gallagher D, Heymsfield SB, Heo M, Jebb SA, Murgatroyd PR, Sakamoto Y. Healthy percentage body fat ranges: an approach for developing guidelines based on body mass index. Am J Clin Nutr 2000; 3: 694-701.

5. Duarte ACG. Avaliação Nutricional: Aspectos Clínicose laboratoriais. São Paulo: Atheneu; 2007.

6. WHO. Obesity: Preventing and Managing the Global Epidemic. Report of a WHO consultation. World Health Organ Tech Rep Ser 2000; 894: i-xii, 1-253.

7. Michels KB, Greenland S, Rosner BA. Does body mass index adequately capture the relation of body composition and body size to health outcomes? Am J Epidemiol 1998; 2: 167-72.
8. Molarius A, Seidell JC, Sans S, Tuomilehto J, Kuulasmaa K. Waist and hip circumferences, and waist-hip ratio in 19 populations of the WHO MONICA Project. Int J Obes Relat Metab Disord 1999; 2: 116-25.

9. Brasil. Pesquisa Nacional Sobre Saúde e Nutrição de 1989. Condições nutricionais da população brasileira: adultos e idosos. Brasília: INAN; 1991.

10. Brasil. Pesquisa de Orçamento Familiar 2002-2003. Análise da Disponibilidade Domiciliar de Alimentos e do Estado Nutricional no Brasil. Rio de Janeiro: Ministério do Planejamento Orçamento e Gestão Instituto Brasileiro de Geografia e Estatística; 2004.

11. Brasil. Ministério da Saúde. PNDS 2006 - Pesquisa Nacional de Demografia e Saúde da Criança e da Mulher. Brasília (DF): Relatório Final; 2008. Disponível em http://bvsms.saude. gov.br/ bvs/pnds/img/relatorio_ final_pnds2006.pdf. [Acessado em 3 de janeiro de 2009]

12. Olinto MTA, Nácul LC, Dias-da-Costa JS, Gigante DP, Menezes AMB, Macedo S. Níveis de intervenção para obesidade abdominal: prevalência e fatores associados. Cad Saúde Pública 2006; 22: 1207-15. 
13. Velásquez-Meléndez G, Pimenta AM, Kac G. Epidemiologia do sobrepeso e da obesidade e seus fatores determinantes em Belo Horizonte (MG), Brasil: estudo transversal de base populacional. Rev Panam Salud Pública 2004; 16: 308-14.

14. Teichmann L, Olinto MTA, Costa JSDd, Ziegler D. Fatores de risco associados ao sobrepeso e a obesidade em mulheres de São Leopoldo, RS. Rev Bras Epidemiol 2006; 9: 360-73.

15. Mendonça CP, Anjos LA. Aspectos das práticas alimentares e da atividade física como determinantes do crescimento do sobrepeso/obesidade no Brasil. Cad Saúde Pública 2004; 20: 698-709.

16. Dallongeville J, Marecaux N, Ducimetiere P, Ferrieres J, Arveiler D, Bingham A et al. Influence of alcohol consumption and various beverages on waist girth and waist-to-hip ratio in a sample of French men and women. Int J Obes Relat Metab Disord 1998; 12: 1178-83.

17. Castanheira M, Olinto MTA, Gigante DP. Associação de variáveis sócio-demográficas e comportamentais com a gordura abdominal em adultos: estudo de base populacional no Sul do Brasil. Cad Saúde Pública 2003; 19: S55-S65.

18. Alves MTSB, Sousa SMPS, Coimbra LC. Atenção à saúde do adulto e da criança no Maranhão. São Luís: EDUFMA; 2009.

19. WHO. Physical status: the use and interpretation of anthropometry. Report of a WHO Expert Committee. World Health Organ Tech Rep Ser 1995; 854: 1-452.

20. Lee ES, Forthofer RN, Lorimer RJ. Analyzing Complex Survey Data. 2th edition. Newbury Park: SAGE; 2006.

21. Stata Corp. Stata Topics Survey Data Analysis. UCLA: Academic Technology Services, Statistical Consulting Group. Disponível em http://www.ats.ucla.edu/stat/ Stata/topics/ Survey.htm. [Acessado em 14 de abril de 2010]

22. Carrasco F, Reyes E, Rimler O, Rios F. Exactitud del índice de masa corporal en la predicción de la adiposidad medida por impedanciometría bioeléctrica. Arch Latinoam Nutr 2004; 54(3): 280-6.

23. Martins IS, Marinho SP. O potencial diagnóstico dos indicadores da obesidade centralizada. Rev Saúde Pública 2003; 37: 760-7.

24. Fonseca MJM, Faerstein E, Chor D, Lopes CS, Andreozzi VL. Associações entre escolaridade, renda e Índice de Massa Corporal em funcionários de uma universidade no Rio de Janeiro, Brasil: estudo Pró-Saúde. Cad Saúde Pública 2006; 22: 2359-67.
25. Monteiro CA, Conde WL, Popkin BM. Independent effects of income and education on the risk of obesity in the Brazilian adult population. J Nutr 2001; 3: S881-S6.

26. Olinto, MTA, Costa JSD, Kac G. Epidemiologia da obesidade abdominal em mulheres adultas residentes no sul do Brasil. ALAN 2007; 4: 349-56.

27. Oliveira EO, Velásquez-Meléndez G, Kac G. Fatores demográficos e comportamentais associados à obesidade abdominal em usuárias de centro de saúde de Belo Horizonte, Minas Gerais, Brasil. Rev Nutr 2007; 20: 361-9.

28. McLaren L, Gauvin L. Neighbourhood level versus individual level correlates of women's body dissatisfaction: toward a multilevel understanding of the role of affluence. J Epidemiol Community Health 2002; 56: 193-9.

29. Blowers LC, Loxton NJ, Grady-Flesser MG, Occhipinti S, Dawe S. The relationship between sociocultural pressure to be thin and body dissatisfaction in preadolescent girls. Eating Behaviors 2003; 4: 229-44.

30. Cattarin J, Williams R, Thomas CM, Thompson KJ. Body image, mood, and televised images of attractiveness: The role of social comparison. Br J Clin Psychol 2000; 19: 220-39.

31. Pope Jr HG, Gruber AJ, Mangweth B, Boreau B, DeCol C, Jouvent $\mathrm{R}$ et al. Body image perception among men in three countries. Am J Psychiatry 2000; 157: 1297-301.

32. Souza LJd, Gicovate Neto C, Chalita FEB, Reis AFF, Bastos DA, Souto Filho JTD et al. Prevalência de obesidade e fatores de risco cardiovascular em Campos, Rio de Janeiro. Arq Bras Endocrinol Metab 2003; 47: 66976 .

33. Han TS, Bijnen FC, Lean ME, Seidell JC. Separate associations of waist and hip circumference with lifestyle factors. Int J Epidemiol 1998; 3: 422-30.

34. Velasquez-Melendez G, Martins IS, Cervato AM, Fornes NS, Marucci MF, Coelho LT. Relationship between stature, overweight and central obesity in the adult population in Sao Paulo, Brazil. Int J Obes Relat Metab Disord 1999; 6: 639-44.

Recebido em: 12/01/2010

Versão final reapresentada em: 08/06/10 Aprovado em: 21/06/10 